Arq. Bras. Med. Vet. Zootec., v.68, n.1, p.233-242, 2016

\title{
Perfil de ácidos graxos e características da carne de bovinos Nelore confinados com diferentes fontes lipídicas protegidas
}

\author{
[Fatty acids and meat characteristics of feedlot Nellore cattle with different \\ protected fat sources] \\ R.S. Barducci ${ }^{1}$, M.C.S. Franzói ${ }^{1}$, L.M.N. Sarti ${ }^{1}$, D.D. Millen ${ }^{2}$, T.C. Putarov ${ }^{1}$, A. Perdigão ${ }^{1}$, \\ C.L. Martins ${ }^{1}$, M.D.B. Arrigoni ${ }^{1}$ \\ ${ }^{1}$ Universidade Estadual Paulista - Botucatu, São Paulo \\ ${ }^{2}$ Faculdade de Zootecnia - Universidade Estadual Paulista - Dracena, SP
}

\begin{abstract}
RESUMO
O objetivo deste trabalho foi avaliar os efeitos da adição de fontes de lipídeos naturais e protegidos da degradação ruminal na dieta de bovinos Nelore confinados sobre características da carne, concentrações de lipoproteínas sanguíneas e perfil de ácidos graxos da gordura do músculo Longissimus dorsi. Foram utilizados 120 bovinos Nelore, não castrados $(366,9 \pm 28,7 \mathrm{~kg})$, com aproximadamente 24 meses. O delineamento experimental foi inteiramente casualizado, com três tratamentos: (CONTR) sem fonte adicional de lipídeo, (GDESP) com fonte de lipídeo natural (torta de algodão) e (GPROT) com fonte de lipídeo protegido rico em ácidos graxos poli-insaturados. Foram utilizadas 24 baias, cinco animais/baia, com oito repetições/tratamento, sendo as baias consideradas unidades experimentais. Foi observada $(\mathrm{P}<0,05)$ interação entre tratamento e dias de mensuração para colesterol e LDL, com maiores valores do tratamento GPROT nos dias 1 e 15, tendo o dia 84 valor intermediário aos demais tratamentos. Observaram-se efeitos de dias de mensuração, em que VLDL e triglicerídeos apresentaram valores crescentes nos dias 15, 84 e 1; enquanto o HDL teve maior valor no dia 1 . Foi observado efeito $(\mathrm{P}<0,05)$ de tratamento para o perfil de ácidos graxos, com diminuição do C14:1, C16:1 e C17:1 e aumento do ácido transvaccênico, C18:2, ácidos graxos poli-insaturados (AGPI) e da relação AGPI: AGMI (ácidos graxos monoinsaturados) para os tratamentos GPROT e GDESP. Adição de lipídios na dieta, independentemente da fonte, promove melhora na composição de ácidos graxos da carne de bovinos Nelore confinados, aumentando a quantidade de ácidos graxos insaturados sem alterar as características qualitativas da carne.
\end{abstract}

Palavras-chave: ácidos graxos insaturados, atributos da carne, lipoproteínas sanguíneas

\begin{abstract}
The objective of this work was to evaluate the effects of adding sources of natural lipids and rumenprotected lipid source rich in polyunsaturated fatty acids to the diet of feedlot Nellore cattle on meat characteristics, blood lipoprotein concentration and fatty acid profile of subcutaneous fat of Longissimus dorsi. One hundred and twenty 24-mo-old Nellore yearling bulls were used (366.9 $\pm 28.7 \mathrm{~kg})$. The experimental design was completely randomized, replicated 8 times (5 bulls/pen), with pens being considered experimental units and consisting of three treatments: (CONTR) without additional source of lipid, (GDESP) with source of natural lipid (cottonseed cake), and (GPROT) with rumen-protected lipid source rich in polyunsaturated fatty acids. Interaction $(P<0.05)$ was observed between treatment and day of measurement for cholesterol and LDL, with values greater GPROT treatment on days 1 and 15, and day 84 intermediate value to the other treatments. Effects $(P<0.05)$ were observed on days of measurement, which showed an increase in VLDL and triglyceride values on days 15, 84 and 1; while HDL had higher values on day 1. Effect $(P<0.05)$ of treatment for the fatty acid profile showed decreased C14:1, C16:1 and C17:1 and increased transvaccênico acid, C18:2, polyunsaturated fatty acids (PUFA)
\end{abstract}

Recebido em 3 de outubro de 2014

Aceito em 29 de junho de 2015

E-mail: robsonbarducci@yahoo.com.br 
and the ratio PUFA:MUFA (monounsaturated fatty acids) for GPROT and GDESP treatments. Additional fat in the diet, regardless of the source, promotes improvement in fatty acid composition of meat from feedlot Nellore cattle, increasing the amount of unsaturated fatty acids without changing the qualitative characteristics of meat.

Keywords: unsaturated fatty acids, attributes of meat, blood lipoproteins.

\section{INTRODUÇ̃̃̃O}

A pecuária nacional vem passando por transformações nos últimos anos, com participação importante do setor de produção de carne, com tendência de constante intensificação por meio do aumento de confinamentos.

Destaca-se a potencialidade do crescimento brasileiro com papel relevante no mercado de produção mundial de carne. O Brasil possui, hoje, o maior rebanho bovino comercial do mundo, é o maior exportador em volume, segundo maior produtor mundial de carne bovina, além de apresentar o terceiro maior consumo interno e quarto consumo per capita. As exportações brasileiras de carne bovina atingiram a marca de US\$ 7,2 bilhões no ano de 2014 , crescimento de 7,7\% em comparação com os US\$ 6,6 bilhões do ano anterior, e volume de 1.099 milhão de toneladas (ABIEC, 2015).

Em consequência dessa evolução da pecuária e da ampla exigência do atual mercado consumidor, maior atenção passou a ser dada aos produtos finais da pecuária de corte. Informações dos atributos intrínsecos da carne passaram a ser pontos importantes, proporcionando dados para avaliar a qualidade do sistema de produção. Sabe-se que a dieta fornecida ao bovino pode alterar a composição da carne, e o seu conhecimento é necessário, já que o valor nutricional da carne como alimento depende da sua composição.

Sendo assim, dietas que forneçam ácidos graxos insaturados são interessantes, pois podem mudar o perfil de ácidos graxos da carne, além de melhorar a qualidade da carne quando fontes lipídicas protegidas da degradação ruminal são oferecidas para bovinos em terminação (Palmquist e Mattos, 2006). Entretanto, são escassos os estudos desenvolvidos nas condições brasileiras, principalmente com animais Nelore que contemplam o uso de fontes de lipídeos naturais e protegidos durante o confinamento. Por isso, são necessários mais estudos relacionados a esse tema, visando à melhoria das características qualitativas da carne.

Assim, o objetivo deste trabalho foi avaliar os efeitos da adição de fontes de lipídeos naturais (coprodutos de algodão) e protegidos (ácidos graxos poli-insaturados) na dieta de bovinos Nelore confinados sobre as características da carne, concentrações de lipoproteínas sanguíneas e perfil de ácidos graxos da gordura.

\section{MATERIAL E MÉTODOS}

Este estudo foi conduzido conforme normas do comitê de ética (nº 34/2011 CEUA) da Faculdade de Medicina Veterinária e Zootecnia, UNESP Universidade Estadual Paulista, Campus de Botucatu, nas instalações do confinamento experimental do Departamento de Melhoramento e Nutrição Animal.

O delineamento experimental utilizado foi inteiramente ao acaso e com três tratamentos: (CONTR) sem fonte adicional de lipídeo, (GDESP) com fonte de lipídeos proveniente de coprodutos do algodão, e (GPROT) com fonte de lipídeo protegido da degradação ruminal rico em ácidos graxos poli-insaturados (Megalac-E®, sais de cálcio de ácido graxo de cadeia longa: Arm \& Hammer, Church \& Dwight Company, QGN, Rio de Janeiro/RJ, Brasil).

Foram utilizados 120 animais machos não castrados da raça Nelore, com peso vivo inicial (PVI) de 366,9 $\pm 28,7 \mathrm{~kg}$, provenientes de recria em sistema de pastejo contínuo (Urochloa brizantha cv. Marandú; oferta total de 3,130kg). $\mathrm{Na}$ chegada ao confinamento, os animais foram pesados (em jejum proporcionado pelo transporte de 16 horas) e distribuídos de acordo com os tratamentos, em que cada tratamento consistiu em oito baias (cinco animais/baia, com $10 \mathrm{~m}^{2} /$ animal e $1 \mathrm{~m}$ de cocho/animal), sendo estas consideradas as unidades experimentais.

As dietas foram formuladas pelo sistema Large Ruminant Nutrition System (LRNS) 1.0.12, nível 
2, sendo adotado protocolo de adaptação gradual às dietas, dividido em três dietas $(55,65$ e $75 \%$ de concentrado), por 14 dias (primeira fornecida por 4 dias e as duas restantes por 5 dias cada), até atingir o nível de concentrado desejado para dieta de terminação $(85 \%)$, no $15^{\circ}$ dia de estudo (Tab. 1). Os animais foram alimentados ad libitum duas vezes ao dia ( $8 \mathrm{~h}$ e $15 \mathrm{~h}$ ) com água constante em bebedouros tipo australiano.

Tabela 1. Composição e conteúdo nutricional das dietas oferecidas aos animais durante o estudo (confinamento)

\begin{tabular}{lccc}
\hline & & Tratamentos $^{(1)}$ & \\
Ingredientes $(\% \mathrm{MS})$ & CONTR & GDESP & GPROT \\
\cline { 2 - 4 } & 15,50 & 14,85 & 15,43 \\
Bagaço de cana cru & 50,29 & 53,12 & 50,62 \\
Silagem grão úmido de milho & 21,37 & 10,08 & 19,14 \\
Polpa Cítrica & 9,43 & - & 9,51 \\
Farelo de amendoim & - & 18,64 & - \\
Torta de algodão & - & - & 1,85 \\
Megalac-E® & 0,95 & 0,98 & 0,99 \\
Ureia & 2,46 & 2,33 & 2,47 \\
Suplemento Mineral ${ }^{(2)}$ & & & 71,00 \\
Conteúdo nutricional ${ }^{(3)}$ & 71,00 & 13,70 & 13,70 \\
MS (\%) & 13,70 & 5,30 & 5,20 \\
Proteína Bruta (\%) & 3,60 & 21,40 & 16,50 \\
Extrato etéreo (\%) & 17,00 & 80,00 & 82,00 \\
FDN (\%) & 80,00 & 1,29 & 1,34 \\
NDT $(\%)$ & 1,29 & 62,00 \\
NEg (Mcal/kg) & 63,00 & 0,00 & 1,06 \\
CNF (\%) & 0,94 & 0,68 & 0,32 \\
Cálcio (\%) & 0,32 & 0,46 & F́sforo (\%)
\end{tabular}

${ }^{(1)}$ CONTR. - dieta controle; GDESP - dieta com gordura proveniente da torta de algodão; GPROT - dieta com adição de fonte de ácidos graxos poli-insaturados protegidos no rúmen. ${ }^{(2)}$ Níveis de garantia: $16,0 \%$ de Ca; $2,4 \%$ de P; 5,9\% de Na; 2,8\% de S; 0,5\% de Mg; $1.680 \mathrm{ppm}$ de Zn; $1.120 \mathrm{ppm}$ de $\mathrm{Mn} ; 560 \mathrm{ppm}$ de Cu; 28ppm de I; 5,6ppm de Se; 8,23ppm de Co e 73.920 UI de vit. A; 1.000 ppm de Monensina. ${ }^{(3)}$ Valores estimados pelo LRNS 1.0.12.

A ingestão de matéria seca (IMS) foi medida para cada baia por meio da pesagem do alimento fornecido diariamente. A pesagem da sobra foi efetuada todos os dias antes do trato da manhã, fazendo-se, posteriormente, a média de ingestão por animal. Os dados de IMS também foram expressos em porcentagem do peso vivo (PV).

Foram feitas pesagens intermediárias a cada 21 dias, jejum de sólidos de 16 horas, para monitorar o ganho de peso diário (GPD) e ajustar a dieta, quando necessário. A conversão alimentar (CA) foi calculada pela divisão da IMS total pelo ganho de PV total durante o experimento, e a eficiência alimentar (EA) foi calculada pela divisão do ganho de PV total pela IMS total durante o período experimental.

Para análises de lipoproteínas do sangue, nos dias 1,15 e 84 de confinamento (primeiro dia de dieta de adaptação, primeiro dia de dieta de terminação e último dia de confinamento, respectivamente), amostras de sangue foram colhidas por meio da punção da veia jugular dos animais (dois animais/baia, totalizando 48 animais) em tubos com vácuo, seguindo a metodologia proposta por Allain et al. (1974). As amostras foram centrifugadas à velocidade de 3.000rpm por 15 minutos, e uma alíquota de $1 \mathrm{~mL}$ de soro foi colocada em dois tubos eppendorf de $0,5 \mathrm{~mL}$, nos quais foi armazenada por no máximo 24 horas até a realização da análise.

Para determinação das concentrações de triglicerídeos, colesterol e lipoproteínas de alta densidade (HDL), foram utilizados kits enzimáticos comerciais (Labtest, Ribeirão Preto, SP, Brasil). 
Os animais foram abatidos com 84 dias de experimento em frigorífico comercial quando alcançaram o PV final (PVF) de $491 \pm 36,4 \mathrm{~kg}$, atendendo às exigências do frigorífico. $\mathrm{O}$ rendimento de carcaça $(\mathrm{RC})$ foi calculado a partir do peso da carcaça quente (PQC) dividido pelo peso vivo final (PVF) do animal. A porcentagem de gordura visceral na carcaça foi calculada pela quantidade $(\mathrm{kg})$ de gordura visceral dividida pelo PCQ. A variável gordura visceral utilizada foi constituída pela gordura de mesentério somada à gordura renal e pélvica.

Após o abate, as carcaças foram mantidas em câmara fria por 24 horas. Ao final do período de resfriamento, foi realizada a medida de $\mathrm{pH}$ final (pH24) das carcaças. As medidas foram obtidas diretamente no músculo, por meio da utilização de um pHgâmetro digital da marca Hanna, modelo HI 8314, com eletrodo de penetração. Durante a desossa, foram coletadas amostras de gordura subcutânea do músculo Longissimus de 48 animais (dois animais/baia), acondicionadas em nitrogênio líquido para análise do perfil de ácidos graxos. Quatro amostras de $2,5 \mathrm{~cm}$ do músculo Longissimus, entre a $10^{\mathrm{a}}$ e $13^{\mathrm{a}}$ costelas de cada animal, foram embaladas a vácuo individualmente e congeladas em freezer ($20^{\circ} \mathrm{C}$ ). As amostras foram utilizadas para análises de cor, perda por cocção (PPC), espessura de gordura subcutânea (EGS), área de olho de lombo (AOL), força de cisalhamento (FC), lipídeos totais e painel sensorial.

Uma amostra de cada animal foi descongelada em geladeira por 24 horas, desembalada, exposta ao ambiente por 20 a 30 minutos para oxigenação da mioglobina e, então, foi determinada a cor da carne mediante leitura em cinco pontos distintos no músculo, utilizando-se o colorímetro portátil Minolta, modelo Chroma Meter CR-410, e a escala do sistema CIELAB, por meio de leituras de refletância da luz em três dimensões: L* (luminosidade), a* (vermelho) e b* (amarelo), segundo metodologia descrita por Honikel (1998).

A análise de PPC foi realizada segundo a metodologia de Honikel (1998). Para a avaliação da FC foram utilizadas as amostras usadas para determinação da $\mathrm{PPC}$, após refrigeração a $4^{\circ} \mathrm{C}$ por 12 horas, seguindo metodologia de Wheeler et al. (1997). As mensurações de EGS foram realizadas com auxílio de paquímetro digital, na porção superior na curvatura do músculo. A medida da AOL foi realizada no músculo por meio de traçado em papel vegetal para posterior avaliação em mesa digitalizadora, modelo MDD 1812 (DIGICOM). As imagens foram analisadas pelo programa SPLAN - Sistema de Planimetria Digitalizador.

Para análise de lipídeos totais foi utilizada a metodologia descrita por Bligh e Dyer (1959). A extração e avaliação dos lipídeos totais foram realizadas de acordo com a metodologia modificada de Hara e Radim (1982), com uso de hexano/isopropanol 3:2 (v/v). Na transesterificação dos ácidos graxos, foi utilizada a metodologia descrita por Christie (1982), com modificações, utilizando solução metanólica de metóxido de sódio.

$\mathrm{Na}$ avaliação da análise sensorial, utilizou-se o método afetivo de aceitação com escala hedônica de nove pontos, com 48 provadores com faixa etária de 20 a 40 anos, de ambos os sexos, seguindo os padrões adotados por Meilgaard et al. (1990). Foram avaliados atributos de aroma, maciez, suculência e sabor.

Para análise estatística, os dados foram inicialmente testados quanto à normalidade $\mathrm{e}$ heterogeneidade de variâncias antes de se proceder à análise de variância, e, quando necessário, os dados foram transformados. Os dados foram analisados utilizando-se o procedimento PROC MIXED do SAS (Statistical..., 2003) e método Satterthwaite de aproximação para determinar os graus de liberdade do denominador para os testes de efeitos fixos.

O modelo estatístico para análises de características de carcaça, perfil de ácidos graxos na gordura, características de cor, físicas, químicas e sensoriais da carne contém os efeitos fixos do tratamento. Dados foram analisados usando-se baia (tratamento) como efeito aleatório. $\mathrm{O}$ modelo estatístico para as lipoproteínas sanguíneas contém os efeitos fixos do tratamento, dias de coleta e interações resultantes. Os dados foram analisados usando-se baia (tratamento) como efeito aleatório e dias como repetição. Estrutura de covariância utilizada foi autoregressivo, que proporcionou o menor critério de informação Akaike e, portanto, o melhor ajuste. Foi utilizado o teste Tukey para 
comparação entre médias e considerados valores significativos de $\mathrm{P}<0,05$.

\section{RESULTADOS}

Para as características de carcaça não foi observado efeito $(\mathrm{P}>0,05)$ de tratamento (Tab. 2).
Quanto aos aspectos qualitativos da carne, não foi observado efeito $(\mathrm{P}>0,05)$ de tratamento sobre as características de cor (Chroma A, Chroma B e Luminosidade), características físicas e químicas $(\mathrm{pH}$, força de cisalhamento (FC), perdas por cocção (PPC), porcentagem de lipídeos na carne), e painel sensorial (sabor, aroma, maciez e suculência) (Tab. 3).

Tabela 2. Características de carcaça de bovinos Nelore confinados suplementados com fontes de lipídeos naturais e protegidos

\begin{tabular}{lccccc}
\multicolumn{1}{c}{ Itens } & \multicolumn{3}{c}{ Tratamentos $^{\mathrm{a}}$} & \multirow{2}{*}{ EPM $^{\mathrm{g}}$} & \multirow{2}{*}{ Valor de P } \\
\cline { 2 - 4 } & CONTR & GDESP & GPROT & & \\
\hline $\mathrm{PCQ}^{\mathrm{b}}, \mathrm{kg}$ & 264,38 & 278,31 & 266,02 & 7,20 & 0,34 \\
$\mathrm{RC}^{\mathrm{c}}, \%$ & 54,25 & 54,50 & 55,00 & 0,04 & 0,35 \\
$\mathrm{GV}^{\mathrm{d}}, \mathrm{kg}$ & 3,17 & 3,64 & 3,34 & 0,16 & 0,13 \\
$\mathrm{GV}^{\mathrm{d}}, \%$ do PCQ & 1,20 & 1,32 & 1,26 & 0,06 & 0,40 \\
$\mathrm{AOL}^{\mathrm{e}}, \mathrm{cm}^{2}$ & 68,98 & 66,98 & 71,80 & 3,13 & 0,32 \\
$\mathrm{EGS}^{\mathrm{f}}, \mathrm{mm}$ & 3,05 & 2,83 & 2,65 & 0,36 & 0,74 \\
\hline
\end{tabular}

${ }^{a}$ CONTR - dieta controle; GDESP - dieta com fonte de lipídeos proveniente de coprodutos do algodão; GPROT dieta com fonte de lipídeo protegido da degradação ruminal, rico em ácidos graxos poli-insaturados. ${ }^{\mathrm{b}} \mathrm{PCQ}$ - peso de carcaça quente; ${ }^{\mathrm{c}} \mathrm{RD}$ - rendimento de carcaça; ${ }^{\mathrm{d}} \mathrm{GV}$ - gordura visceral; ${ }^{\mathrm{e}} \mathrm{AOL}$ - área de olho de lombo; ${ }^{\mathrm{f}} \mathrm{EGS}$ espessura de gordura subcutânea; ${ }^{\mathrm{g}}$ Erro padrão médio.

Tabela 3. Características de cor, físicas e químicas e sensoriais da carne de bovinos Nelores confinados suplementados com fontes de lipídeos naturais e protegidos

\begin{tabular}{|c|c|c|c|c|c|}
\hline \multirow[b]{2}{*}{ Características de cor } & \multicolumn{3}{|c|}{ Tratamentos $^{\mathrm{a}}$} & \multirow[b]{2}{*}{$\mathrm{EPM}^{\mathrm{b}}$} & \multirow[b]{2}{*}{ Valor de P } \\
\hline & CONTR & GDESP & GPROT & & \\
\hline \multicolumn{6}{|l|}{ Cor } \\
\hline Chroma A & 18,52 & 18,27 & 18,49 & 0,52 & 0,93 \\
\hline Chroma B & 4,01 & 4,19 & 4,26 & 0,39 & 0,89 \\
\hline Luminosidade & 35,63 & 35,37 & 35,99 & 0,49 & 0,68 \\
\hline \multicolumn{6}{|l|}{ Físicas e químicas } \\
\hline $\mathrm{pH}$ & 6,36 & 6,27 & 6,42 & 0,11 & 0,66 \\
\hline Força de cisalhamento, $\mathrm{kg}$ & 5,23 & 5,88 & 5,29 & 0,56 & 0,67 \\
\hline Perdas por Cocção, \% & 19,12 & 19,34 & 19,93 & 1,22 & 0,89 \\
\hline Lipídeos (Carne), \% & 0,65 & 0,78 & 0,75 & 0,11 & 0,66 \\
\hline \multicolumn{6}{|l|}{ Painel Sensorial } \\
\hline Sabor & 8,25 & 7,62 & 6,75 & 0,55 & 0,18 \\
\hline Aroma & 6,62 & 7,5 & 7,5 & 0,59 & 0,5 \\
\hline Maciez & 6,25 & 7,12 & 6,87 & 0,39 & 0,28 \\
\hline Suculência & 7,25 & 7,5 & 6,25 & 0,49 & 0,56 \\
\hline
\end{tabular}

${ }^{a}$ CONTR - dieta controle; GDESP - dieta com fonte de lipídeos proveniente de coprodutos do algodão; GPROT dieta com fonte de lipídeo protegido da degradação ruminal, rico em ácidos graxos poli-insaturados; ${ }^{b}$ Erro padrão médio.

Em relação aos valores de concentração de lipoproteínas sanguíneas (Tab. 4), foi observada interação $(\mathrm{P}<0,05)$ entre tratamento e dia de mensuração para o colesterol e LDL (Fig. 1 e 2), em que o tratamento GPROT apresentou maior concentração no dia 1 e 15 em relação ao demais tratamentos; ao passo que, no dia 84, apresentou valor intermediário. 


\section{Barducci et al.}

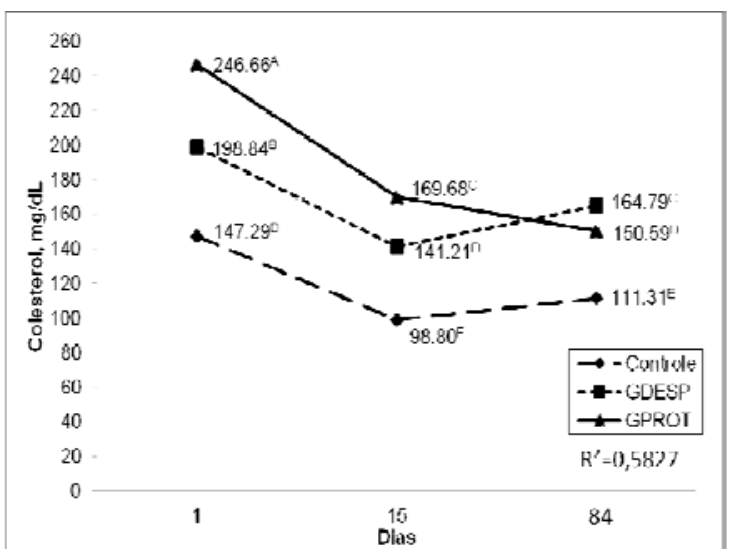

Figura 1. Concentração de colesterol $(\mathrm{mg} / \mathrm{dL})$ no sangue de bovinos Nelore confinados suplementados com fontes de lipídeos naturais e protegidos. Médias com diferentes sobrescritos diferem pelo teste Tukey $(\mathrm{P}<0,05)$.

Também se observou efeito $(\mathrm{P}<0,05)$ de dias de mensuração para HDL, VLDL e triglicerídeos (Tab. 4). A concentração de HDL no dia 1 apresentou maior valor que os dias 15 e 84, não

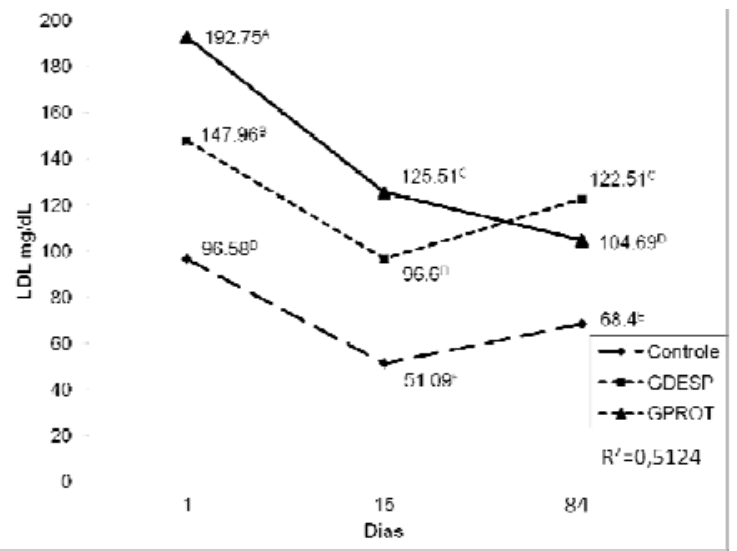

Figura 2. Concentração de LDL (mg/dL) no sangue de bovinos Nelore confinados suplementados com fontes de lipídeos naturais e protegidos. Médias com diferentes sobrescritos diferem pelo teste Tukey $(\mathrm{P}<0,05)$.

havendo diferença entre os dias 15 e 84. As concentrações de VLDL e triglicerídeos apresentaram maiores valores no dia 15 , seguido em ordem decrescente para o dia 84 e 1 .

Tabela 4. Valores de concentração de lipoproteínas sanguíneas de bovinos Nelore confinados suplementados com fontes de lipídeos naturais e protegidos

\begin{tabular}{|c|c|c|c|c|c|c|c|c|c|c|}
\hline \multirow[b]{2}{*}{ Item $(\mathrm{mg} / \mathrm{dL})$} & \multicolumn{3}{|c|}{${\text { Tratamentos }(\mathrm{T})^{\mathrm{d}}}^{\mathrm{d}}$} & \multicolumn{3}{|c|}{ Dias (D) } & \multirow{2}{*}{$\mathrm{EPM}^{\mathrm{h}}$} & \multicolumn{3}{|c|}{ Valor de $\mathrm{P}$} \\
\hline & CONTR & GDESP & GPROT & 1 & 15 & 84 & & $\mathrm{~T}$ & $\mathrm{D}$ & INT $^{\mathrm{i}}$ \\
\hline Coles & 118,13 & 168,28 & 188,98 & 197,6 & 135,6 & 142,2 & 3,17 & $<0,01$ & $<0,01$ & $\mathrm{X}$ \\
\hline $\mathrm{HDL}^{\mathrm{e}}$ & 43,48 & 43,46 & 45,24 & $49,45 \mathrm{a}$ & $41,63 b$ & $41,11 b$ & 1,61 & 0,67 & $<0,01$ & NS \\
\hline $\mathrm{LDL}^{\mathrm{f}}$ & 72,02 & 122,36 & 140,98 & 145,8 & 91,07 & 98,53 & 3,44 & $<0,01$ & $<0,01$ & $\mathrm{X}$ \\
\hline $\mathrm{VLDL}^{\mathrm{g}}$ & 2,62 & 2,46 & 2,76 & $2,38 \mathrm{c}$ & $2,87 \mathrm{a}$ & $2,59 b$ & 0,09 & 0,17 & $<0,01$ & NS \\
\hline Triglicerídeos & 13,12 & 12,29 & 13,78 & $11,89 \mathrm{c}$ & $14,34 \mathrm{a}$ & $12,96 \mathrm{~b}$ & 0,45 & 0,17 & $<0,01$ & NS \\
\hline
\end{tabular}

Médias na mesma linha com diferentes sobrescritos diferem-se pelo teste de Tukey $(\mathrm{P}<0,05)$; ${ }^{\mathrm{d}} \mathrm{CONTR}-$ dieta controle; GDESP - dieta com fonte de lipídeos proveniente de coprodutos do algodão; GPROT - dieta com fonte de lipídeo protegido da degradação ruminal, rico em ácidos graxos poli-insaturados; ${ }^{\mathrm{e}} \mathrm{HDL}$ - lipoproteínas de alta densidade; ${ }^{\mathrm{f}} \mathrm{LDL}$ - lipoproteínas de baixa densidade; ${ }^{\mathrm{g}} \mathrm{VLDL}$ - lipoproteína de muito baixa densidade; ${ }^{\mathrm{h}}$ Erro padrão médio; ${ }^{\mathrm{i}} \mathrm{INT}=$ Interação; NS - Não Significativo $(\mathrm{P}>0,05) ; \mathrm{x}$ - interação entre tratamentos e dias.

Para o perfil de ácidos graxos, foi observado efeito $(\mathrm{P}<0,05)$ de tratamento, com diminuição do ácido mirístico (C14:1), ácido palmítico (C16:1) e ácido heptadecenoico (C17:1), e aumento do ácido transvaccênico, ácido linoleico
(C18:2), ácidos graxos poli-insaturados (AGPI) e da relação AGPI: AGMI (ácidos graxos monoinsaturados) para os tratamentos GPROT e GDESP (Tab. 5). 
Perfil de ácidos graxos...

Tabela 5. Perfil de ácidos graxos na gordura de bovinos Nelore confinados suplementados com fontes de lipídeos naturais e protegidos

\begin{tabular}{|c|c|c|c|c|c|}
\hline \multirow{2}{*}{ Ácidos Graxos (g/100g de gordura) } & \multicolumn{3}{|c|}{ Tratamentos $^{\mathrm{c}}$} & \multirow{2}{*}{$\mathrm{EPM}^{\mathrm{m}}$} & \multirow[b]{2}{*}{ Valor de $\mathrm{P}$} \\
\hline & CONTR & GDESP & GPROT & & \\
\hline $\mathrm{AGCC}^{\mathrm{d}}$ & 0,200 & 0,170 & 0,250 & 0,020 & 0,14 \\
\hline C14:0 & 4,060 & 3,910 & 4,090 & 0,170 & 0,72 \\
\hline C14:1 & $1,030 \mathrm{a}$ & $0,810 \mathrm{~b}$ & $0,840 \mathrm{~b}$ & 0,060 & 0,04 \\
\hline C15:0 & 1,060 & 0,970 & 0,990 & 0,050 & 0,48 \\
\hline C16:0 & 24,470 & 25,100 & 24,480 & 0,440 & 0,51 \\
\hline C16:1 & $3,840 \mathrm{a}$ & $3,200 \mathrm{~b}$ & $3,250 \mathrm{~b}$ & 0,130 & $<0,01$ \\
\hline $\mathrm{C} 17: 0$ & 1,350 & 1,270 & 1,260 & 0,030 & 0,07 \\
\hline $\mathrm{C} 17: 1$ & $0,800 \mathrm{a}$ & $0,620 \mathrm{~b}$ & $0,620 \mathrm{~b}$ & 0,020 & $<0,01$ \\
\hline C18:0 & 15,350 & 17,170 & 16,980 & 0,700 & 0,15 \\
\hline C18:1 & 1,260 & 1,630 & 0,370 & 0,990 & 0,24 \\
\hline $\mathrm{TVA}^{\mathrm{e}}$ trans- $10,11,12$ & $4,970 \mathrm{~b}$ & $6,310 \mathrm{a}$ & $6,940 \mathrm{a}$ & 0,330 & $<0,01$ \\
\hline C18:2 & $1,570 \mathrm{~b}$ & $2,240 \mathrm{a}$ & $3,060 \mathrm{a}$ & 0,110 & $<0,01$ \\
\hline CLA $^{\mathrm{f}}$ cis- 9 , trans- 11 & 0,690 & 0,740 & 0,760 & 0,040 & 0,49 \\
\hline C18:3 & 0,160 & 0,150 & 0,160 & 0,020 & 0,55 \\
\hline $\mathrm{C} 20: 4$ & 0,020 & 0,015 & 0,020 & 0,002 & 0,27 \\
\hline Ômega-6 & 0,003 & 0,002 & 0,003 & 0,001 & 0,33 \\
\hline Ômega-3 & 0,160 & 0,150 & 0,160 & 0,010 & 0,59 \\
\hline Ômega-6:ômega-3 & 0,020 & 0,020 & 0,020 & 0,010 & 0,67 \\
\hline $\mathrm{AGS}^{\mathrm{g}}$ & 46,66 & 48,75 & 48,18 & 0,86 & 0,22 \\
\hline $\mathrm{AGI}^{\mathrm{h}}$ & 52,16 & 50,18 & 50,69 & 0,80 & 0,22 \\
\hline MUFA $^{\mathrm{i}}$ & 50,08 & 47,69 & 48,36 & 0,81 & 0,12 \\
\hline PUFA $^{\mathrm{j}}$ & $2,07 \mathrm{~b}$ & $2,48 \mathrm{a}$ & $2,33 \mathrm{a}$ & 0,08 & $<0,01$ \\
\hline PUFA:MUFA & $0,042 b$ & $0,052 \mathrm{a}$ & $0,048 \mathrm{a}$ & 0,003 & $<0,01$ \\
\hline $\mathrm{AGNI}^{1}$ & 1,19 & 1,07 & 1,13 & 0,11 & 0,75 \\
\hline
\end{tabular}

Médias na mesma linha com diferentes sobrescritos diferem-se pelo teste de Tukey $(\mathrm{P}<0,05)$; ${ }^{\mathrm{c}} \mathrm{CONTR}-$ dieta controle; GDESP - dieta com fonte de lipídeos proveniente de coprodutos do algodão; GPROT - dieta com fonte de

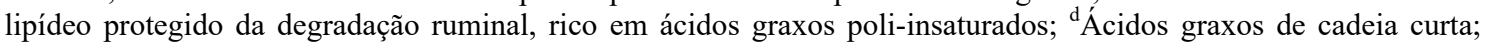
eÁcido transvaccênico; ${ }^{\mathrm{f}}$ Ácido linoleico conjugado; ${ }^{\mathrm{g}}$ Ácidos graxos saturados; ${ }^{\mathrm{h}}$ Ácidos graxos insaturados; ${ }^{\mathrm{i}}$ Ácidos graxos monoinsaturados; ${ }^{\mathrm{j}}$ Ácidos graxos poli-insaturados; ${ }^{1}$ Ácidos graxos não identificados; ${ }^{\mathrm{m}}$ Erro padrão médio.

\section{DISCUSSÃO}

O decréscimo do $\mathrm{pH}$ é processo bioquímico necessário para que o músculo do animal abatido se transforme em carne. Com as alterações postmortem, esse parâmetro deve chegar a 5,4, duas a oito horas após a sangria, quando se inicia o rigor mortis (Sobrinho, 2006). Entretanto, a velocidade de queda, bem como o $\mathrm{pH}$ final da carne após 24 horas, é muito variável, e vários fatores determinam a velocidade da queda, o início e duração do rigor-mortis. A resposta do animal a cada fator depende da espécie, peso, idade, sexo e resistência do animal aos agentes estressantes, bem como o estado emocional do próprio (Roça, 2000).

No entanto, se devido a deficiência de glicogênio, o pH permanecer após 24 horas acima de 6,2, tem-se o indício de carne DFD (dark, firm, dry); problema causado pelo estresse crônico antes do abate, que esgota os níveis de glicogênio. Há evidências de que o principal fator para o aparecimento seja manejo inadequado antes do abate, que conduz à exaustão física do animal. Os valores de $\mathrm{pH}$ 
estão pouco acima desse valor, no entanto, não houve influência negativa na aparência visual da carne no que diz respeito a cor e luminosidade fator de qualidade que o consumidor mais aprecia no momento da compra (Sobrinho, 2006). Semelhantemente a esses resultados, Fernandes et al. (2009) observaram valores de pH 6,16 para tourinhos Nelore terminados em confinamento, o qual os relaciona à reatividade dos animais durante o manejo pré-abate. Bovinos confinados são mais susceptíveis ao estresse do que os criados a pasto, o que pode contribuir para que a redução do $\mathrm{pH}$ após o resfriamento não seja efetiva, e, portanto, animais submetidos a condições de estresse apresentam maior gasto do glicogênio muscular e menor produção de ácido lático, responsável pela redução do pH (Gregory, 1998).

Ainda quanto às características qualitativas da carne, os valores de AOL e PPC estão dentro da normalidade (Rubiano et al., 2009). Entretanto, os valores de EGS e FC ficaram fora da normalidade, fator que torna a carne menos macia independentemente da suplementação lipídica (Oliveira, 2000). Outros autores relataram valores de $\mathrm{FC}$ próximos deste resultado (Andrade et al., 2011; Li et al., 2010), que pode ter ocorrido pelo encolhimento da fibra muscular. A deposição de gordura subcutânea pode ter sido prejudicada devido à sazonalidade do período experimental e o tempo de duração que os animais permaneceram confinados, dado que os mesmos não apresentavam EGS necessária para $\mathrm{o}$ abate, que favorece $\mathrm{o}$ resfriamento mais rápido das massas musculares, provocava o encurtamento dos sarcômeros e, consequentemente, maior FC.

Outro fator importante relacionado aos altos valores para a FC está relacionado à raça Nelore, que possui maiores quantidades de ligações cruzadas do colágeno, além de maior ação de enzimas, como a calpastatina, que inibem o amaciamento da carne. Rubensam et al. (1998) relataram que valores elevados de FC são comuns em zebuínos, característica possivelmente associada à maior atividade da enzima calpastatina, que possui efeito inibidor sobre as calpaínas, responsáveis pela proteólise post-mortem (amaciamento da carne). Abularach et al. (1998) concluíram que participação crescente do Bos indicus no genótipo resulta em carne menos macia.
Outro método para analisar maciez da carne é através do painel sensorial, que ficou acima da média 5 na escala hedônica de 9 pontos, o que pode considerar a carne macia apesar de a FC estar acima da normalidade. Não apenas na maciez, como também todos os outros atributos avaliados no painel sensorial (sabor, aroma e suculência) ficaram acima da média 5, não havendo diferença significativa entre os tratamentos. O aroma, sabor e suculência, quando avaliados na escala não estruturada de nove pontos, foram próximos aos atributos característicos da carne bovina e superior ao encontrado por Manço (2006) em bovinos da raça Nelore com dois anos de idade.

A queda do teor total de colesterol e LDL no tratamento GPROT ao longo do confinamento mostrou que a alimentação com grande quantidade de poli-insaturados auxilia em sua diminuição. $\mathrm{O}$ ácido linoleico possui habilidade de reduzir a concentração de LDL-colesterol, semelhantemente a medicamentos hipocolesterolêmicos. Lipídeos séricos enriquecidos com ácidos graxos poliinsaturados ocupam mais espaço dentro das partículas de lipoproteínas, consequentemente, menores quantidades de moléculas de éstercolesterol serão encontradas no interior das LDL, portanto, não se reduziria os teores de LDL e sim os teores de colesterol em cada partícula de LDL (Spritz e Mishkel, 1969).

As taxas de lipídeos totais observadas normalmente em bovinos comerciais variam de 1 a 3\% na Bélgica (Cuvelier et al., 2006), na Itália (Cifuni et al., 2004), em Portugal (Alfaia et al., 2007) e na Espanha (Serra et al., 2008); e de 4 a 5\% na Argentina (Garcia et al., 2008) e Nova Zelândia (Purchas e Zou, 2008), e acima de 10\% no Japão (Purchas e Zou, 2008). Neste trabalho, as taxas de gordura no Longissimus dorsi foram inferiores à maioria dos valores relatados na literatura. Dessa maneira, no perfil lipídico talvez possa implicar a diminuição na proporção de ácidos graxos saturados e aumento nos ácidos graxos poli-insaturados. Entre os aumentos dos ácidos graxos avaliados, pode-se destacar o ácido linoleico conjugado (CLA). Maior ingestão de CLA proporciona efeitos positivos à saúde, que incluem redução na deposição de gordura corporal, alteração na partição de nutrientes, efeitos antidiabéticos, redução no desenvolvimento de aterosclerose, melhoria da 
mineralização dos ossos e do sistema imunológico (Bauman et al., 1999).

Além dos fatores alimentares que influenciaram positivamente na composição em ácidos graxos da carne, a utilização de animais da raça Nelore teve grande influência, pois esse tipo de animal apresenta maior ação da enzima delta 9dessaturase (Fernandes et al., 2009), que contribui para a transformação de ácidos graxos saturados em insaturados e a transformação do ácido graxo transvaccênico em CLA. Como observado, os ácidos graxos transvaccênico, ácidos graxos poli-insaturados e relação PUFA:MUFA dos tratamentos GDESP e GPROT apresentaram valores maiores; resultado que mostra a importância de fornecer dieta rica em ácidos graxos poli-insaturados, não importando a fonte de lipídeo. Dessa forma, o que irá diferenciar a escolha de um ou outro será o seu custo.

\section{CONCLUSÃO}

Adição de lipídios na dieta, independentemente da fonte, promove melhora na composição de ácidos graxos da carne de bovinos Nelore confinados, aumentando a quantidade de ácidos graxos insaturados sem alterar as características qualitativas da carne.

\section{REFERÊNCIAS}

ABULARACH, M.L.; ROCHA, C.E.; FELÍCIO, P.E. Características de qualidade do contrafilé (m. L. dorsi) de touros jovens da raça Nelore. Ciênc. Tecnol. Aliment., v.18, p.205-210, 1998.

ALFAIA, C.M.M.; CASTRO, M.L.F.; MARTINS, S.I. et al. Effect of slaughter season on fatty acid composition, conjugated linoleic acid isomers and nutritional value of intramuscular fat in Barrosã-PDO veal. Meat Sci., v.75, p.44-52, 2007.

ALLAIN, C.C.; POON, L.S.; CHAN, C.S.G. et al. Enzymatic determination of total serum cholesterol. Clin. Chem., v.120, p.470-475, 1974.

ANDRADE, E.N.; COSTA, Q.P.B.; GIRÃO, L.V.C. et al. Parâmetros de qualidade da carne de bovinos nelore submetidos a diferentes temperaturas de cocção. In: CONGRESSO BRASILEIRO DE MEDICINA VETERINÁRIA, 38. 2011, Florianópolis/SC. Anais... Florianópolis/SC: SBMV, 2011. CD ROM.

BAUMAN, D.E.; BAUMGARD, L.H.; CORL, B.A. Biosynthesis of conjugates linoleic acid in ruminants. J. Anim. Sci., v.77, p.1-15, 1999.
BLIGH, E.G.; DYER, W.J. A rapid method of total lipid extraction and purification. Can. J. Biochem. Physiol., v.37, p.911. 1959.

CHRISTIE, W.W. Simple produce for rapid transmethy lation of glycerolipids and cholesteryl esters. J. Lipid Res., v.23, p.1-4, 1982.

CIFUNI, G.F.; NAPOLITANO, F.; RIVIEZZI, A.M. et al. Fatty acid profile, cholesterol content and tenderness of meat from Podolian young bulls. Meat Sci., v.67, p.289-297, 2004.

CUVELIER, C.; CABARAUX, J.F.; DUFRASNE, I. et al. Comparison of composition and quality traits of meat from young finishing bulls from Belgian Blue, Limousin and Aberdeen Angus breeds. Meat Sci., v.74, p.522-531, 2006.

EXPORTAÇÕES brasileiras de carne bovina, 2015. ABIEC, 2015. Disponível em: $<$ http://www.abiec.com.br/download/Anual-2015250515.pdf $>$. Acessado em: 20 jul. 2015.

FERNANDES, A.R.M.; SAMPAIO, A.A.M.; HENRIQUE, W. et al. Composição em ácidos graxos e qualidade de carne de tourinhos Nelore e Canchim alimentados com dietas à base de cana-de-açúcar e dois níveis de concentrado. Rev. Bras. Zootec., v.38, p.328-337, 2009.

GARCIA, P.T.; PENSEL, N.A.; SANCHO, A.M. et al. Beef lipids in relation to animal breed and nutrition in Argentina. Meat Sci., v.79, p.500-508, 2008.

GREGORY, N.G. Animal welfare and meat science. Cambridge: University Press, 1998. 289p.

HARA, A.; RANDIN, N.S. Lipid extraction of tissues with a low toxicity solvent. Anal. Biochem,. v.90, p.420-426, 1982.

HONIKEL, K.O. Reference methods for the assessment of physical characteristics of meat. Meat Sci., v.49, p.447-457, 1998.

LI, C.B.; ZHOU, G.H.; XU, X.L. Dynamical Changes of Beef Intramuscular Connective Tissue and Muscle Fiber during Heating and their Effects on Beef Shear Force. Food Bioprocess Technol., v.3, p.521-527, 2010.

MANÇO, M.C.W. Características físico-químicas, sensoriais e higiênicas da carne bovina em duas classes de maturidade e sob influência da maturação. 2006. 124f. Tese (Doutorado em Zootecnia) Universidade Estadual Paulista, Botucatu, SP.

MEILGAARD, M.; CIVILlE, G.V.; CARR, B.T. Sensory evaluation techniques. Boca Raton: CRC Press, 1990. 281p.

OLIVEIRA, A. L. Maciez da carne bovina. Cad. Tec. Vet. Zootec., n.33, p.7-18, 2000. 


\section{Barducci et al.}

PALMQUIST, D.L.; MATTOS, W.R.S. Metabolismo de lipídeos. In: BERCHIELLI, T.T.; PIRES, A.V.; OLIVEIRA, S.G. (Ed.s). Nutrição de ruminantes. Jaboticabal: Funep, 2006. p. 287-310.

PURCHAS, R.W.; ZOU, M. Composition and quality differences between the longissimus and infraspinatus muscles for several groups of pasture-finished cattle. Meat Sci., v.80, p.470-479, 2008.

ROÇA, R. O. Tecnologia da carne e produtos derivados. Botucatu: UNESP/Faculdade de Ciências Agronômicas, 2000. 202p.

RUBENSAM, J.M.; FELÍCIO, P.E.; TERMIGNONI, C. Influência do genótipo Bos indicus na atividade de calpastatina e na textura da carne de novilhos abatidos no sul do Brasil. Ciênc. Tecnol. Aliment., v.18, 1998. Disponível em: <http://dx.doi.org/10.1590/S010120611998000400009>.

RUBIANO, G.A.G.; ARRIGONI, M.B.; MARTINS, C.M. et al. Desempenho, características de carcaça e qualidade da carne de bovinos superprecoces das raças Canchim, Nelore e seus mestiços. Rev. Bras. Zootec., v.38, p.2490-2498, 2009.
SERRA, X.; GUERRERO, L.; GUARDIA, M.D. et al. Eating quality of young bulls from three Spanish beef breed-production systems and its relationships with chemical and instrumental meat quality. Meat Sci., v.79, p.98-104, 2008.

SOBRINHO, A.G.S. (3.ed). Criação de ovinos. Jaboticabal: Funep, 2006. 302p.

SPRITZ, N.; MISHKEL, M.A. Effects of dietary fats on plasma lipids and lipoproteins; a hypothesis for the lipid-lowering effect of unsaturated fatty acids. J. Clin. Invest., v.48, p.78-86, 1969.

STATISTICAL analysis system - SAS user's guide: statistics. 5.ed. Cary: SAS Institute, 2003. 846p.

WHEELER, T.L.; SHACKELFORD, S.D.; JOHNSON, L.P. et al. A comparison of WarnerBratzler shear force assessment within and among institutions. J. Anim. Sci., v.75, p.2423-2432, 1997. 